\title{
Magnetic Noise Reduction of In-Wheel Permanent Magnet Synchronous Motors for Light-Duty Electric Vehicles
}

\author{
Pedram Asef $1,2, * \mathbb{D}$, Ramon Bargallo ${ }^{2} \mathbb{D}$ and Andrew Lapthorn ${ }^{3} \mathbb{D}$ \\ 1 Department of Mechanical Engineering, Centre for Automotive Engineering, University of Surrey, \\ Guildford GU2 7XH, UK \\ 2 Department of Electrical Engineering, Universitat Politècnica de Catalunya-BarcelonaTech, 08019 Barcelona, \\ Spain; ramon.bargallo@upc.edu \\ 3 Department of Electrical and Computer Engineering, University of Canterbury, Christchurch 8041, \\ New Zealand; andrew.lapthorn@canterbury.ac.nz \\ * Correspondence: p.asef@surrey.ac.uk
}

Received: 22 November 2019; Accepted: 23 February 2020; Published: 25 February 2020

\begin{abstract}
This paper presents study of a multi-slice subdomain model (MS-SDM) for persistent low-frequency sound, in a wheel hub-mounted permanent magnet synchronous motor (WHM-PMSM) with a fractional-slot non-overlapping concentrated winding for a light-duty, fully electric vehicle applications. While this type of winding provides numerous potential benefits, it has also the largest magnetomotive force (MMF) distortion factor, which leads to the electro-vibro-acoustics production, unless additional machine design considerations are carried out. To minimize the magnetic noise level radiated by the PMSM, a skewing technique is targeted with consideration of the natural frequencies under a variable-speed-range analysis. To ensure the impact of the minimization technique used, magnetic force harmonics, along with acoustic sonograms, is computed by MS-SDM and verified by $3 \mathrm{D}$ finite element analysis. On the basis of the studied models, we derived and experimentally verified the optimized model with $5 \mathrm{dBA}$ reduction in A-weighted sound power level by due to the choice of skew angle. In addition, we investigated whether or not the skewing slice number can be of importance on the vibro-acoustic objectives in the studied WHM-PMSM.
\end{abstract}

Keywords: electric vehicle; electric motor; magnetic noise; vibration; multi-slice subdomain model

\section{Introduction}

Among all main sources of acoustic noise, such as mechanical and aerodynamic, the magnetic source can be very critical in many applications (transport systems, aerospace, electric vehicle (EV) motors, etc.), particularly in high-torque motors, where a large number of poles and a thinner stator yoke always result in a medium mechanical stiffness. This induces magnetic vibration due to magnetic forces which consist of Maxwell and magnetostrictive forces. Fractional-slot concentrated windings (FSCW) are very popular because of many remarkable enhancements during recent years in terms of manufacturability and modularity [1], increased flux weakening performance [2], fault tolerance features [3], and short-end windings [4,5]. However, the acoustic noise needs special attention because of the small number of slots per pole per phase q, which causes large magnetomotive force (MMF) distortion due to low-order harmonics in the spatial distribution of the radial forces in comparison to other traditional permanent magnet (PM) machines [6-8].

The first attempt to reduce magnetic vibrations and acoustic noise produced in the electrical machines via Maxwell forces has been modeled in Reference $[9,10]$. 
In recent research work, there are several studies to present the impact of various machine design considerations on magnetic noises with considering variable-speed and natural frequencies conditions as follows.

J. Le Besnerais et al. studied a multi-objective optimization on a fast analytical model of a variable-speed induction machine which calculates both motor performances and sound power level of electromagnetic origin. The model has been coupled to a Non-dominating Sorting Genetic Algorithm (NSGA-II) in order to perform global constrained optimizations with respect to several objectives (e.g., noise level, efficiency, and material cost) [11]. In another work [12], the authors present a fast simulation tool for the variable-speed magnetic noise emitted by induction machines, based on fully analytical models. On the basis of these models, they derive and experimentally validate an analytical expression for magnetic vibrations due to slotting reluctance harmonics, confirming the prime importance of slot combination in magnetic noise radiation. Finally, a database that efficiently replaces the old empirical rules for slot combination numbers and helps in designing quiet induction motors has been proposed which can be built for other power ranges. In Reference [13], an analytical model is investigated that is developed for surface mounted permanent magnet machines with skewed slots via the subdomain technique and the multislice method. In another study [14], the authors study a step skewed rotor which is applied to the design to reduce electro-magnetic exciting force so that both reduction of vibration and noise and cost-effectiveness of development can be achieved for interior permanent magnet synchronous machines (IPMSMs). Furthermore, the influence of vibration-based forces and noises due to pulse width modulation (PWM) supply in induction machines have been studied in Reference $[15,16]$.

\subsection{Research Gap and Original Contributions}

The vast majority of recent works focused on noise and vibration analyses in electric machines caused by mechanical and aerodynamic sources, such as variability in design, texturing the blades, material features, manufacturing, bearings (sleeve, fluid, and balls), fan selection (radial or axial), etc. The electromagnetic source of noise and vibration (known as e-NVH) has been mainly studied based on empirical principles, like relative permeance (which fails to provide accurate predictions, while a variable-speed-range analysis is needed in traction electric motors, reported in Reference [17-19]), and some studies have attempted to use semi-analytical and numerical approaches, such as semi-finite element analysis (FEA) for e-NVH computations. Among them, only a few have reduced the noise and vibration of the electric motor using skewing technique for the electric vehicle applications.

Highly motivated by the research gap, the researchers have proposed a semi-analytical-based approach to compute the e-NVH of the motor under a variable speed range analysis condition with consideration of natural frequencies, and herein, the skewing technique along with various slice numbers on both stator and rotor parts significantly reduced the e-NVH of the in-wheel electric motor for the light-duty electric passenger vehicle. Natural frequency mapping of the motor used in EV application allow the engineers to evaluate the motor's performance under different driving conditions, such as accelerating and braking. Additionally, the achievements are validated by experimental measurements using the manufactured in-wheel motor. Thereby, the numerical and experimental investigation of the in-wheel PMSM under a variable-speed-range analysis is done for the first time.

This paper investigates the impact of a skewing technique to minimize the vibration level power and audible magnetic noise on a wheel hub-mounted permanent magnet synchronous motor (WHM-PMSM) using a multi-slice subdomain model (MS-SDM) with use of non-overlapping FSCW for a variable-speed-range analysis. With respect to the importance of MMF distortion of the FSCW used, the results of both initial and optimized WHM-PMSM are presented. The skewing angle for a wide-range of skew rate has been expressed by stator slot pitch between 0.8-1.2. We investigate whether or not the skewing slice number is significant to the vibro-acoustic objectives in the studied WHM-PMSM. Although many empirical rules have been established for correctly carrying out the skewing of stator and rotor, as to limit the maximum audible magnetic noise level radiated from 
the WHM-PMSM, none of these empirical rules have considered the stator's natural frequencies or variable-speed-analysis. The literature contains papers which have been recently published using empirical-based approaches to choose skewing angle [20-25] and numerical-based studies to minimize vibration and noise $[21,26]$ without considering the natural frequencies, as well as variable-speed analysis in some cases. This study demonstrates how these important considerations can change the choice of skewing angle. In addition to acoustic-based optimization, the electromagnetic performance of the motor, such as torque, cogging torque, and back-electromotive force(back-EMF) parameters, are defined as optimization constraints. We have derived the best skewing rate and slice number in order to reduce the vibration level power and maximum audible magnetic noise, while the electromagnetic performance remained constant. Finally, the multi-slice subdomain model (MS-SDM) optimized WHM-PMSM has numerically (3D-FEA) and experimentally verified the achievements for a fully electric vehicle car, in which the WHM-PMSM's placement in the drivetrain is presented in Figure 1. The battery and ultracapacitor are the primary and secondary energy sources of the drivetrain, respectively.

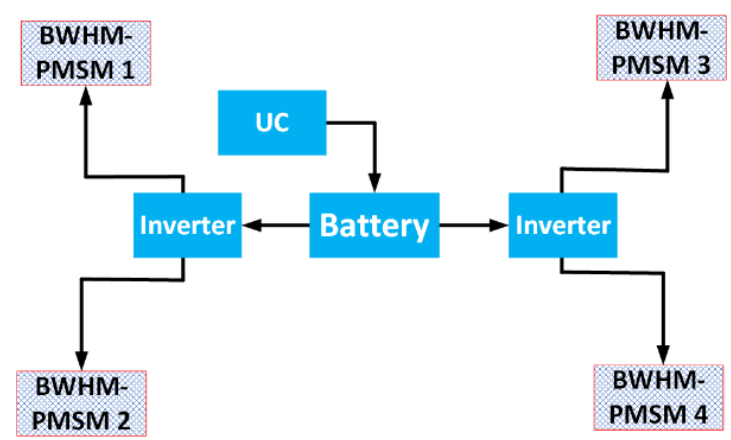

Figure 1. Simplified drivetrain scheme for the light-duty fully electric vehicle. BWHM = brushless wheel hub-mounted; PMSM = permanent magnet synchronous motor; UC = ultra-capacitor.

\subsection{Paper's Organization and Presentation}

The paper is organized as follows. The introduction section brings some insights of the existing efforts on the analytical, semi-analytical, and numerical methods to compute the noise and vibration in the electric motors. In Section 2, the analytical expressions of the PMSM related to the electromagnetic noise and vibration, based on the MS-SDM method is given. Section 3 discusses both the numerical and graphical findings toward the objective that is noise and vibration reduction using skewing technique. Figure 1 shows the WHM-PMSM in the powertrain. Figures 2-7 demonstrate several important electro-magnetic-based behaviors of WHM-PMSM, in which the magnetic noise is targeted on the MS-SDM simulation study results. In Figure 2, the radial component of electromagnetic force is shown using MS-SDM. Figure 3 presents the impact of various skew angles of the stator and rotor on both the $\mathrm{x}$-axis magnetic force and maximum sound power. Additionally, the effect of the skewing technique on the magnitude of the maximum sound power level with respect to the impactful modal contribution of the WHM-PMSM's structure is presented in Figure 4. Figures 5 and 6 reinforce the outcome of the numerical variable speed noise results by a $1 / 3$ octave, A-weighted acoustic noise spectrum, and spectrum where the resonances are considered. Section 4 validates the achievements using FEA and experimental measurement of the manufactured in-wheel PMSM. Figures 8 and 9 verifies the MS-SDM using 3D FEA, and Figures 10 and 11 validate the numerical-based results by experimental tests. Last, the conclusion offers the highlights and main achievements of the work.

\section{Analytical Vibro-Acoustic Modelling Using Multi-Slice Subdomain Model (MS-SDM) for Wheel Hub-Mounted Permanent Magnet Synchronous Motor (WHM-PMSM)}

Magnetic noise phenomenon consists of magnetostrictive and Maxwell forces. To calculate magnetic noise natural frequencies, the Fourier development of the PM is necessary by a permeance/ 
MMF model. This study has to consider the stator and rotor MMF; however, the rotor MMF is much smaller. The transient magnetic field for low frequency can be given by Maxwell's equation:

$$
\left\{\begin{array}{c}
\nabla \times \frac{1}{\sigma} \nabla \times H_{j}+\frac{\partial B_{j}}{\partial t}=0 \\
\nabla \cdot B_{j}=0
\end{array},\right.
$$

where $H \mathrm{j}$ is magnetic field strength of the $j$ th subdomain slice, $B \mathrm{j}$ is magnetic flux density of each subdomain slice $j$ th, $E$ is electric field strength, and $\sigma$ is electric conductivity. The skewing angle depending on the magnitude of the field can be discretized as follows [7]:

$$
b_{\text {skew }}=\frac{1}{\theta_{\text {skew }}} \int_{-\theta_{\text {skew }} / 2}^{\theta_{\text {skew }} / 2} \hat{B}_{j} \cos \left(n \theta+n \theta_{\text {skew }}^{\prime}+\omega t\right) d \theta_{\text {skew }}^{\prime}
$$

where $\hat{B}$ is the magnitude of the field, $n$ denotes spatial order, $\omega$ is the pulsation, and $\theta_{\text {skew }}$ is the skew angle.

To compute the radial component of the force, $F_{\mathrm{x}}$ and $F_{\mathrm{y}}$ are defined based on the cylindrical coordinate system [7]:

$$
\left\{\begin{array}{l}
F_{x j}=\int_{s} p_{r j}(\theta, z) \cos \theta-p_{\theta j}(\theta, z) \sin \theta \\
F_{y j}=\int_{s} p_{r j}(\theta, z) \sin \theta+p_{\theta j}(\theta, z) \cos \theta
\end{array}\right.
$$

where both radial forces, which are basically produced by the stator and rotor winding harmonic magnetic field at $j$ th subdomain slice [7], are:

$$
\left\{\begin{array}{c}
p_{r j}(\theta, z)=\frac{1}{2 \mu_{0}}\left(B_{r j}^{2}(\theta, z)-B_{\theta j}^{2}(\theta, z)-B_{z j}^{2}(\theta, z)\right) \\
p_{\theta j}(\theta, z)=\frac{B_{\theta j}(\theta, z) \cdot B_{r j}(\theta, z)}{\mu_{0}}
\end{array} .\right.
$$

Regarding the Maxwell stress tensor Equations (1)-(3), the radial magnetic force, as a major source of magnetic vibration and as a function of both space and time, can be defined as:

$$
\begin{aligned}
& \vec{F}_{r j}=\frac{1}{2 \mu_{0}}\left(B_{r j}^{2}-B_{t j}^{2}\right)=\frac{1}{2 \mu_{0}}\left(\left[\frac{1}{r} \frac{\partial A_{z g j}}{\partial t}\right]^{2}-\left[\frac{-\partial A_{z g j}}{\partial r}\right]^{2}\right), \\
& \vec{F}_{c}=m \omega^{2} r
\end{aligned}
$$

where $A_{\text {zgj }}$ is the vector potential in the middle of air-gap for the $j$ th subdomain slice, $F_{\mathrm{rj}}$ is the radial magnetic force for the $j$ th subdomain slice, $B_{\mathrm{rj}}$ and $B_{\mathrm{tj}}$ are the radial and tangential components of magnetic flux density in the middle of air-gap $j$ th subdomain slice, $\theta$ is the mechanical angular position, $n$ is the spatial harmonic order, $k$ is the time harmonic order, $\omega$ is the angular velocity, $r$ is the radius of the rotor, and $m$ is the mass of rotor. $F_{\mathrm{c}}$ is the centrifugal force.

Assuming that the stiffness and mass of the model are constant, when the machine is considered as time invariant, all the displacement and deformation should be calculated at each node of the generated meshes. Thereupon, the non-linear vibration of the $j$ th subdomain slice in the WHM-PMSM is [7]:

$$
\left\{F_{j}(t)\right\}=[M]\{\ddot{x}(t)\}+[C]\{\dot{x}(t)\}+[K]\{x(t)\} .
$$

Hence, the sound wave of the WHM-PMSM with a small amplitude is propagating for each $j$ th subdomain slice, which is a simplistic but useful idealistic homogeneous medium as:

$$
\frac{\partial^{2} p_{j}}{\partial x^{2}}+\frac{\partial^{2} p_{j}}{\partial y^{2}}+\frac{\partial^{2} p_{j}}{\partial z^{2}}=\frac{1}{c^{2}} \frac{\partial^{2} p_{j}}{\partial t^{2}} .
$$


The maximum sound power radiated for the $j$ th subdomain slice by the WHM-PMSM due to magnetic vibrations, which can be written as [11,12]:

$$
W_{p j}\left(f_{\max }\right)=\sum_{j=1,2, \ldots, m} \frac{1}{2} Z_{0_{j}} S_{c_{j}} \sigma_{m j}(f)\left\langle v_{m \omega j}^{2}\right\rangle v_{m \omega j},
$$

where $Z_{0 \mathrm{j}}$ denotes the air acoustic impedance, $S_{\mathrm{cj}}$ is the WHM-PMSM frame area at $j$ th slice, and $\sigma_{\mathrm{mj}}$ is the modal radiation factor at $j$ th slice. The maximum sound power level $W_{\omega j}$ at $j$ th subdomain slice [15] can be computed using:

$$
W_{\omega j}\left(f_{\max }\right)=10 \log _{10}\left(\sum_{j} \frac{W_{p j}\left(f_{\max }\right)}{10^{-12}}\right) .
$$

The natural frequencies of the stator circumferential mode have been chosen only as even values due to the stator and rotor pole numbers both being even. Thus, the Maxwell exciting force harmonics are produced only as even spatial orders due to symmetry. By assimilating, the stator to an equivalent ring has been taken into account. The breathing mode natural frequency $f_{0}$ can be defined as [16]:

$$
f_{0}=\frac{1}{2 \pi a} \sqrt{\frac{E_{s}}{K_{f_{s} j} \Delta_{m} \rho_{s j}}},
$$

where $a$ is the stator mean radius, which applies $h_{\mathrm{f}}$ as the frame width, $K_{\mathrm{fsj}}$ is the stator stacking factor at $j$ th slice, $\rho_{\mathrm{sj}}$ is the stator stack mass density at $j$ th slice, and $\Delta_{\mathrm{m}}$ denotes the increasing mass because of the winding and teeth.

Following considerations and assumptions are made for the vibro-acoustic modeling using MS-SDM of WHM-PMSM with skewing:

1. Both stator and rotor are subjected to find the optimal angle of skew at each $j$ th subdomain slice.

2. End effects are neglected because FSCW with non-overlapping configuration have very short end-windings at the studied WHM-PMSM.

3. Leakage flux in axial direction is ignored.

4. Main slotting magnetic forces wavenumbers and frequencies are considered.

5. The range of slice number $j$ is 20 which means one slice per each $5 \mathrm{~mm}$, in case of $100 \mathrm{~mm}$ stack length.

6. Variable speed analysis is targeted for the electromagnetic-acoustic performance of the WHM-PMSM.

7. The magnetic flux density along the axial axis is applied using 2D FEA for a higher accuracy.

8. The demagnetization characteristics of the permanent magnets are considered non-linear.

9. The analysis is done under load condition.

10. Natural frequencies are considered at variable speed analysis for the WHM-PMSM.

11. The saturation effect on the magnetic field is not considered by MS-SDM simulation (more details can be found in Reference $[7,13,26])$.

\section{Results and Discussion}

The results and discussion section include the electro-magnetic impact of various skewing angles on the vibration and acoustic results with special attention on the audible magnetic noise and vibration computations. The input design data of the studied WHM-PMSM for the simulation is presented first. Table 1 indicates this data in three individual sections, geometrical, electrical, and magnetic. The FE analysis is done using the potential vector method with the total time steps of 180, the number of elements of 544,289, and the number of nodes of 163,013. Each design case was simulated using 
nonlinear 3D FEA method. For both the initial and optimized designs, the PMs are made of NdFeB-N48 with a remanent flux density of $1.2 \mathrm{~T}$, and the back irons are made of isotropic M400-50A non-orientated magnetic lamination steel with $7650 \mathrm{~kg} \cdot \mathrm{m}^{-3}$. The elastic characteristics of the lamination is $E_{\mathrm{x}}=215$ $\mathrm{MPa}, E_{\mathrm{y}}=215 \mathrm{MPa}$, and $E_{\mathrm{z}}=80 \mathrm{MPa}$, besides, shear modulus is $G_{\mathrm{xy}}=82.7 \mathrm{MPa}, G_{\mathrm{yz}}=2 \mathrm{MPa}$, and $G_{\mathrm{zx}}=2 \mathrm{Mpa}$; the mechanical properties of the material used can be seen in Table 1. All the following sections are based on 3D FEA unless specifically indicated otherwise.

Table 1. Input data of the studied WHM-PMSM.

\begin{tabular}{|c|c|c|}
\hline Parameters & Values & Units \\
\hline \multicolumn{3}{|c|}{ Geometrical } \\
\hline$R_{r i} / R_{r o}$ & $217 / 230$ & $\mathrm{~mm}$ \\
\hline$R_{s i} / R_{s o}$ & $115 / 209.5$ & $\mathrm{~mm}$ \\
\hline$S_{w}$ & 15 & $\mathrm{~mm}$ \\
\hline$\delta_{g}$ & 0.6 & $\mathrm{~mm}$ \\
\hline$Q_{s} / 2 P$ & $36 / 40$ & - \\
\hline$\alpha_{p}$ & 0.55 & - \\
\hline$S P$ & 0.9 & - \\
\hline \multicolumn{3}{|c|}{ Electrical } \\
\hline$m$ & 3 & - \\
\hline$N_{\mathrm{c}}$ & 80 & turns \\
\hline$J_{\mathrm{rms}}$ & 1.2206 & - \\
\hline$I_{\mathrm{rms}}$ & 4.899 & $\mathrm{~A} / \mathrm{mm}^{2}$ \\
\hline Number of parallel circuit per phase & 2 & - \\
\hline Slot/pole/phase & 0.3 & - \\
\hline$\lambda_{\mathrm{d}}$ & 1703.9381 & $\mathrm{mVs}$ \\
\hline Line-to-line inductance (d-axis) & 937.8789 & $\mathrm{mH}$ \\
\hline \multicolumn{3}{|c|}{ Magnetic } \\
\hline$\mu_{0}$ & $4 \pi \times 10^{-7}$ & $\mathrm{H} / \mathrm{m}$ \\
\hline$\mu_{\mathrm{s}}$ & 2500 & - \\
\hline$\mu_{\mathrm{PM}}$ & 1.05 & - \\
\hline$\mu_{\text {air }}$ & 1 & - \\
\hline$B_{\mathrm{rm}}$ & 1.2 & $\mathrm{~T}$ \\
\hline$H_{k}$ & $891 \times 10^{3}$ & $\mathrm{~A} / \mathrm{m}$ \\
\hline Magnet permeance per half pole & 0.1437 & $\mu \mathrm{Wb} / \mathrm{At}$ \\
\hline
\end{tabular}

Figure 2 illustrates the maximum radial magnetic force variation from Equations (1), (3) and (5) with and without centrifugal force consideration. As presented in the graph, the produced total $(j=20)$ radial forces $F_{\mathrm{r}}$ from the inner stator excited coils, $F_{\mathrm{c}}$, due to its mechanical essence, has a clockwise vector direction (based on the right's hand law) because of the body's inertia with a uniform distribution. Both the radial and centrifugal forces have a distribution with a radial vector direction and have an overlapped influence on each other. A summation of them, leading up to the resultant blue curve in this graph, indicates the total (or actual) radial force on the rotor. 


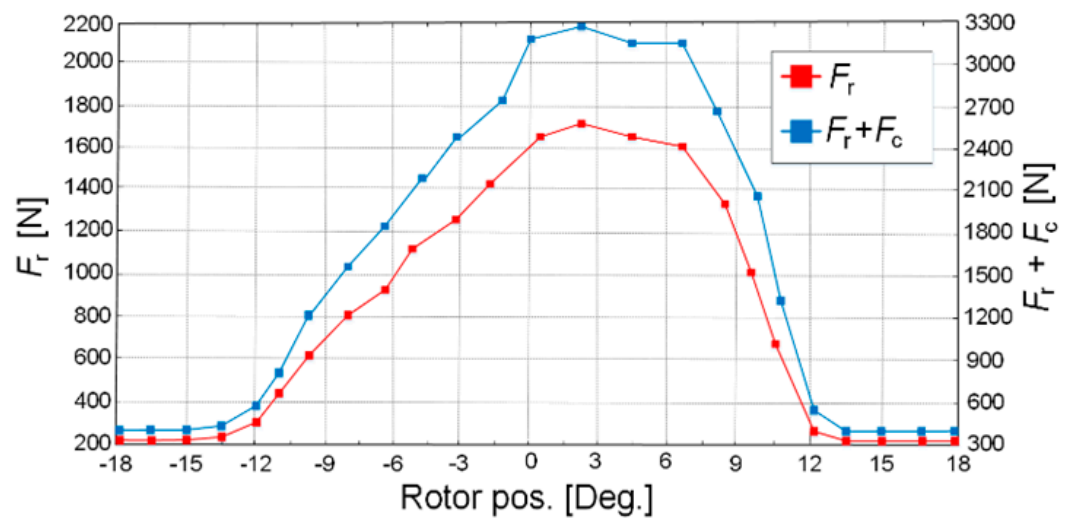

Figure 2. Maximum radial force (normal and centrifugal components) of the wheel hub-mounted permanent magnet synchronous motor (WHM-PMSM) under 4.9A root mean square (RMS) excitation current with $30^{\circ}$ rotation.

The influence of various skew angles (as variables) is studied in the stator/ rotor, slot/ magnet pitch factors unit. Each single slot pitch factor, equal to $10^{\circ}$, from $0.8-1.2$ (due to electromagnetic capability of the studied PMSM) on the $x$-axis component of the magnetic force $F_{\mathrm{x}}$ and A-weighted maximum sound power level $W_{\mathrm{p}, \max }$ (shown in Figure 3) was calculated at variable speeds from $N_{\min }=15 \mathrm{rpm}$ to $N_{\max }=1000 \mathrm{rpm}$. To find the best possible angle for each part (stator and rotor), this computation evaluates the impact of each variable on the objectives with respect to each other. Each graph is also a function of the number of skew slices. Figure 3a presents two main regions (red and blue dashed areas) in which the red area is where the stator skew obtains the highest rate, between 1 to 1.2 , whereas the rotor skew rate is lower. This area provides a higher level of $W_{p, \max }$, between 92.4 to $92.5 \mathrm{dBA}$. The lowest $W_{\mathrm{p}, \max }$ (optimal area) is reached when the highest rate of rotor skew angle (including magnets), and smaller stator angle skew were chosen (92.2 dBA). Comparing to the initial skew angle, which is analytically calculated (black arrow), an enhancement of approximately $1 \mathrm{dBA}$ has been achieved. The analytical MS-SDM method used is based on the average flux density in 2D along the axial direction. Additionally, this graph (Figure 3a) depicted the skew model by $j=20$ slices. Figure $3 b$ illustrates the optimal area as the same as Figure 3 a with ratings of 1.2 and 0.8 for the rotor and stator. However, a larger $F_{\mathrm{x}}$ with skew slice of 20 in comparison with lower slice numbers, which produces consequently higher outputs, such as torque. Figure $3 c$ demonstrates how the odd number (15) of the skew slots again decreases $F_{\mathrm{x}}$ by nearly $100 \mathrm{~N}$. In this configuration, $W_{\mathrm{p}}$ has been enhanced by $1.5 \mathrm{dBA}$ when compared to the initial model. As presented in Figure 3d, higher $F_{\mathrm{x}}$ has been produced by maximum value of $874 \mathrm{~N}$, when an even number (20) of skew slice has been used. Therefore, the importance of the skew slice number on $F_{\mathrm{x}}$ and its harmonics are considerable with respect to the number of stator slots and rotor magnets (or slots, in case of other machine topologies) which can be an even or odd variable), has significant influence on the sound power level as function of speed. This figure helps in identifying number proportionally, although, it could not directly reduce $W_{\mathrm{p}}$.

Figure 4 reinforces the idea that the structural contribution of the WHM-PMSM skewing technique based on various range of skew angles, which is basically a structural-focused analysis of structural modes that are involved in airborne acoustic noise radiation of the studied PMSMs. Additionally, the acoustic noise level enhancement through cancelling the effect of a given structural mode to quantify the modal contribution to magnetic noise is targeted. Figure $4 \mathrm{a}$ indicates the magnetic noise magnitude as function of speed, where the maximum radiated A- weighted magnetic noise of $91 \mathrm{dBA}$, can be seen at $124 \mathrm{rpm}$, however, this quantity is reduced using the optimized model (shown in Figure 4b) by $16 \mathrm{dBA}$. The magnetic noise increases proportionally with the speed, and thus, the maximum sound power of 110 and $91.04 \mathrm{dBA}$ can be reported for both the initial and optimized models. Although the force harmonic modes of $(4,0)$ and $(8,0)$ over the rotational speed range have contributed widely, the overall $W_{\mathrm{p}}$ amplitude is decreased for the optimum skew angles, as shown in Figure $4 \mathrm{~b}$. 

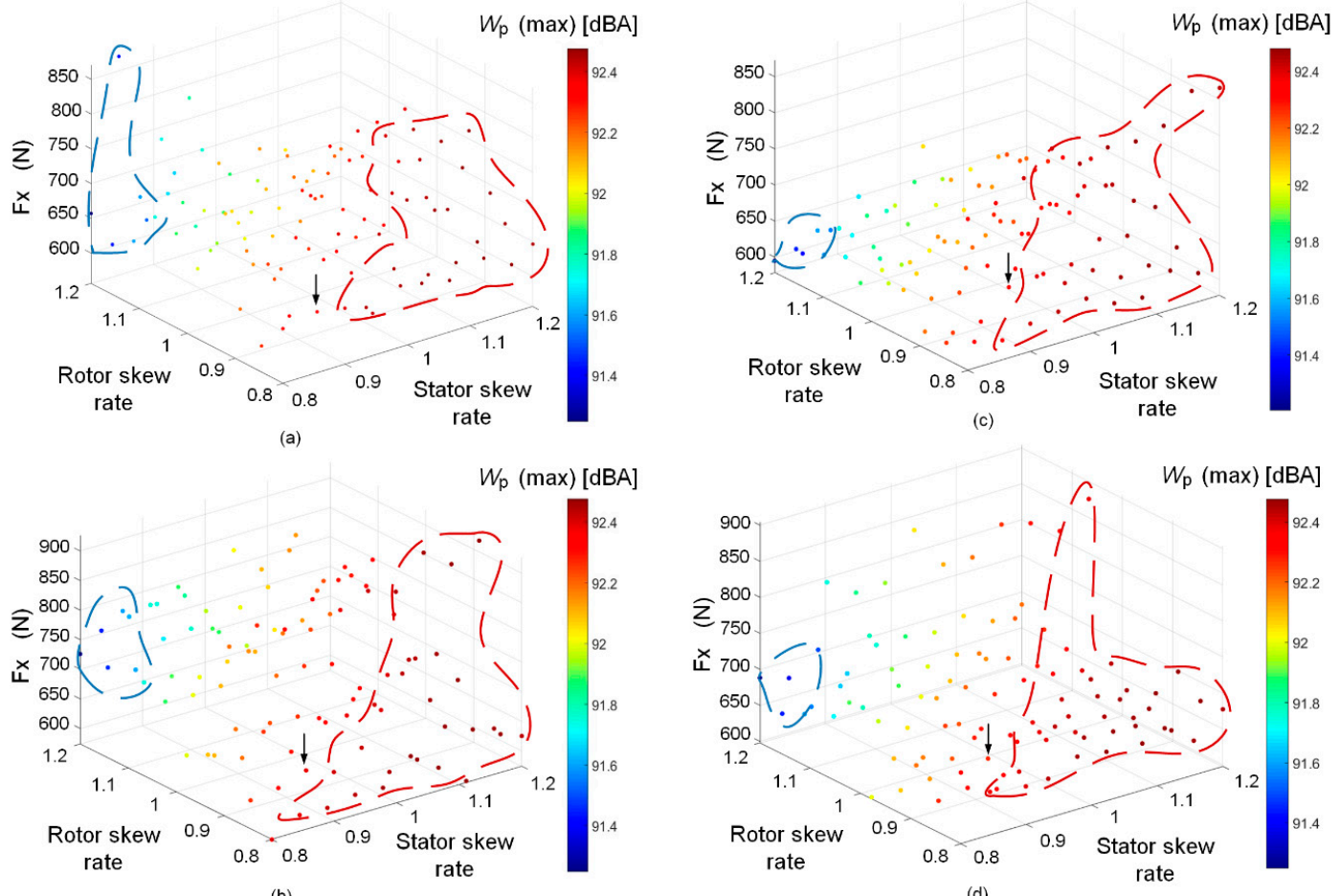

(d)

Figure 3. Objective-variable-based variation of stator and rotor skew angle versus $F_{\mathrm{x}}$ and $L_{\mathrm{p}, \max }$ as function of skew slice number (a) 5 slices, (b) 10 slices, (c) 15 slices, and (d) 20 slices.

Table 2 presents the main amplitudes of the MMF harmonics on both stator and rotor cores. As presented when the skew angles increase, the MMF harmonics (higher MMF harmonics results in higher force harmonics) of both cores are changing significantly. Higher rate of rotor skew angle brings a considerably lower MMF harmonic; however, a higher rate of stator skew angle has resulted in higher MMF harmonics and, finally, higher Wp. Moreover, the choice of best possible skew angles relies on other two important factors, resonance effect and electromagnetic capability. Hence, the final optimum values of both stator and rotor skew angles is provided through a trade-off. The fundamental harmonic $(h=1)$ has its highest value at 1.2 rotor skew rate, which decreased slightly when the skew angle is decreased. The major harmonic orders, such as 3rd, 5th, and 7th, have shown lower harmonics, while the skew angle rates lower. The values are in per unit for a better comparison.

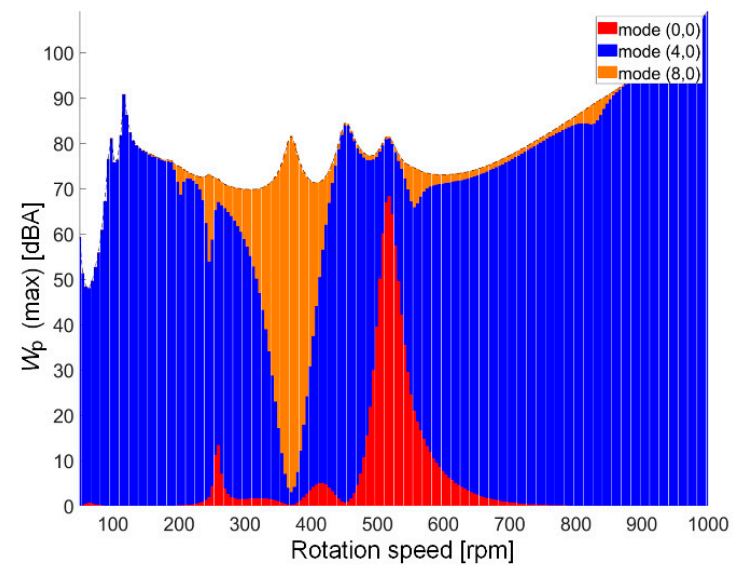

(a)

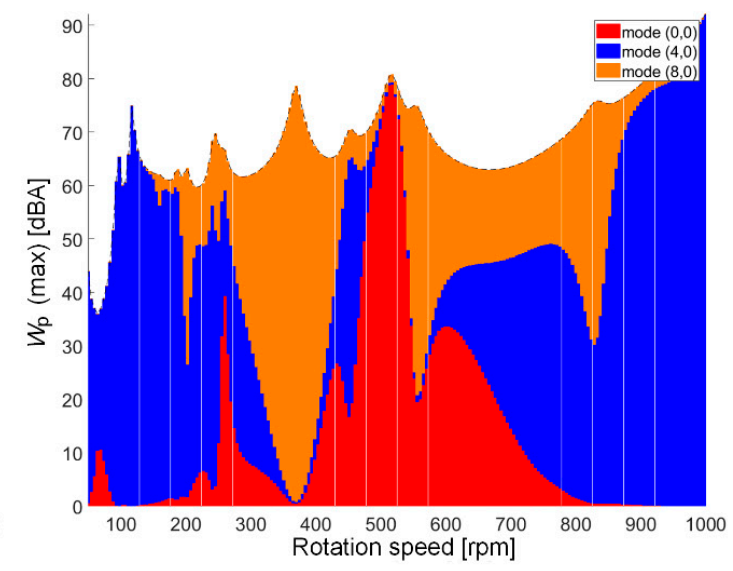

(b)

Figure 4. Modal contribution of the machine's structure on the magnetic noise (sound power), listed as: (a) Initial and (b) Optimized WHM-PMSMs. 
Table 2. Magnetomotive force (MMF)harmonics of different skew angle rates for the rotor core.

\begin{tabular}{cccccccc}
\hline Skew Rates/h. Order & $\mathbf{0 . 6}$ & $\mathbf{0 . 7}$ & $\mathbf{0 . 8}$ & $\mathbf{0 . 9}$ & $\mathbf{1 . 0}$ & $\mathbf{1 . 1}$ & $\mathbf{1 . 2}$ \\
\hline 1 & 0.83 & 0.84 & 0.89 & 0.91 & 0.92 & 0.96 & 0.99 \\
3 & 0.71 & 0.69 & 0.67 & 0.66 & 0.61 & 0.56 & 0.53 \\
5 & 0.47 & 0.46 & 0.44 & 0.41 & 0.41 & 0.38 & 0.37 \\
7 & 0.38 & 0.37 & 0.34 & 0.35 & 0.33 & 0.33 & 0.33 \\
9 & -0.25 & -0.25 & -0.26 & -0.24 & -0.27 & -0.27 & -0.28 \\
11 & -0.3 & -0.29 & -0.33 & -0.34 & -0.3 & -0.29 & -0.29 \\
13 & -0.22 & -0.21 & -0.23 & -0.23 & -0.22 & -0.21 & -0.21 \\
15 & 0.1 & 0.09 & 0.11 & 0.04 & 0.05 & 0.04 & 0.02 \\
17 & 0.23 & 0.19 & 0.18 & 0.21 & 0.2 & 0.2 & 0.19 \\
19 & 0.29 & 0.29 & 0.25 & 0.23 & 0.25 & 0.23 & 0.2 \\
21 & -0.06 & -0.04 & -0.04 & -0.05 & -0.02 & -0.01 & 0 \\
23 & -0.12 & -0.1 & -0.11 & -0.08 & -0.07 & -0.04 & -0.01 \\
25 & -0.09 & -0.07 & -0.07 & -0.05 & -0.04 & -0.04 & -0.04 \\
27 & 0.07 & 0.01 & 0.01 & 0 & 0.01 & 0 & 0 \\
29 & 0.12 & 0.12 & 0.08 & 0.08 & 0.13 & 0.09 & 0.07 \\
31 & 0.07 & 0.08 & 0.07 & 0.04 & 0.02 & 0.02 & 0.01 \\
\hline
\end{tabular}

Figure 5 illustrates the one third octave A-weighted acoustic noise spectrum for the initial (Figure 5a) and optimized (Figure 5b) models. As presented, the maximum sound power bars based on the human's normal audition for each range of frequencies are reduced using the skewing technique. The radiated magnetic noise at rated speed of $150 \mathrm{rpm}$ on both initial and optimized PMSMs has obtained 78.08 and $62.67 \mathrm{dBA}$, respectively. Note that A-weighting was defined by IEC 61672-1:2013, which enables one to convert a physical quantity of acoustic pressure (in $\mathrm{dB}$ ) into a psychoacoustic quantity (in $\mathrm{dBA}$ ) that is supposed to better quantify how loud a noise is perceived.

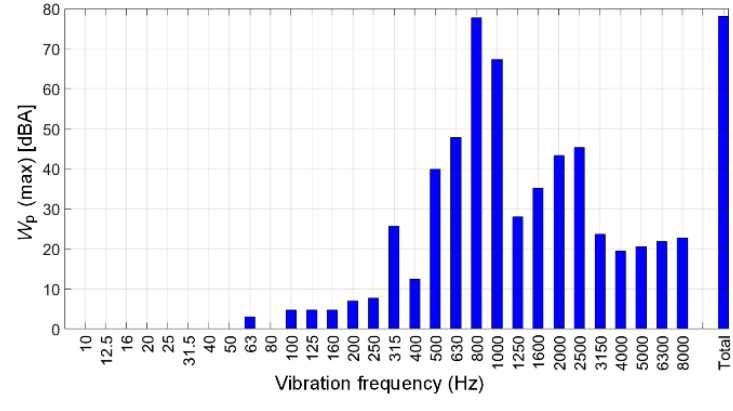

(a)

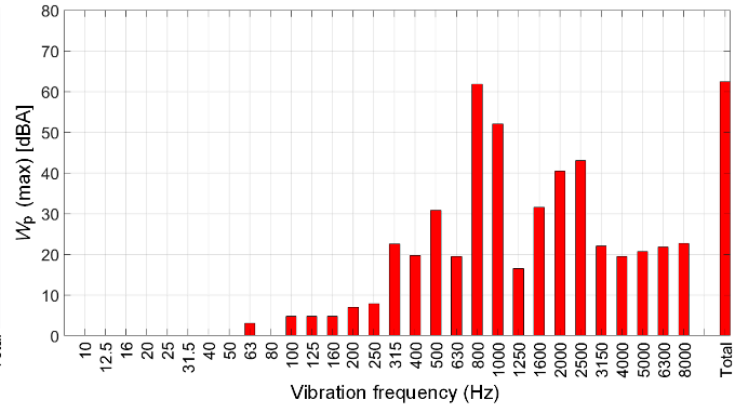

(b)

Figure 5. One third octave A-weighted acoustic noise spectrum, as listed: (a) Initial, (b) Optimized WHM-PMSM.

Figure 6 presents an A-weighted maximum magnetic noise spectrogram of the optimum skew angles of rotor and stator $12^{\circ}$ and $8^{\circ}$ under a variable speed range. The spectrogram is evaluated in the post processing stage, in which the electromagnetically-excited radial vibration illustration identifies whether the vibration resonances of variable speed of the WHM-PMSM due to magnetic forces occur during the operation or not. The designed WHM-PMSM allows to operate under $150 \mathrm{rpm}, 50 \mathrm{~Hz}$, while it is a motor. Figure 6 presents that the first resonance happens at $185 \mathrm{~Hz}$ and $356 \mathrm{rpm}$ which is shown between the grey dashed line in the modal structural modes of $(2,0)$ and $(3,0)$. The main slotting magnetic forces wavenumbers of $\mathrm{r}=0,4$, and 8 are involved in the machine, where the magnetic forces wavenumbers by $\mathrm{r}=\left(2 \times \mathrm{h}_{r}+1\right) \times \mathrm{p}+\varepsilon_{s} \times \mathrm{k}_{s} \times \mathrm{Q}_{s}+\varepsilon_{r} \times \mathrm{p}$ is calculated in which the frequency is $\mathrm{f}=\left(2 \times \mathrm{h}_{r}+1+\varepsilon_{r}\right) \times \mathrm{f}_{s}+\mathrm{r} \times \mathrm{f}_{s} / \mathrm{p}$, where $k_{\mathrm{s}}$ is an integer involved in permeance Fourier series, and $\pm \mathcal{E}$ varies between -1 to 1 . There are two main resonances marked as 1 and 2 , which have occurred at $172 \mathrm{~Hz}$ and $516 \mathrm{rpm}$, as well as $185 \mathrm{~Hz}$ and $554 \mathrm{rpm}$. In addition, the WHM-PMSM rotates 
in the flux weakening condition at $430 \mathrm{rpm}$ which is indicated by the red dashed line. Afterwards, the largest $736 \mathrm{rpm}, 488 \mathrm{~Hz}$. Note that the first resonance at structural mode $(2,0)$ happens when the electrical frequency of the traveling force wave of wavenumber $r$ matches with the circumferential mode natural frequency, where 2 is the rank of the circumferential deflection, and 0 denotes the rank of the longitudinal deflection in the structural mode. To evaluate all the natural frequencies of the WHM-PMSM with rated speed of $15-150 \mathrm{rpm}(0$ to $50 \mathrm{~Hz})$, the radial velocity in unit of dBA should be computed for over $5000 \mathrm{~Hz}$.

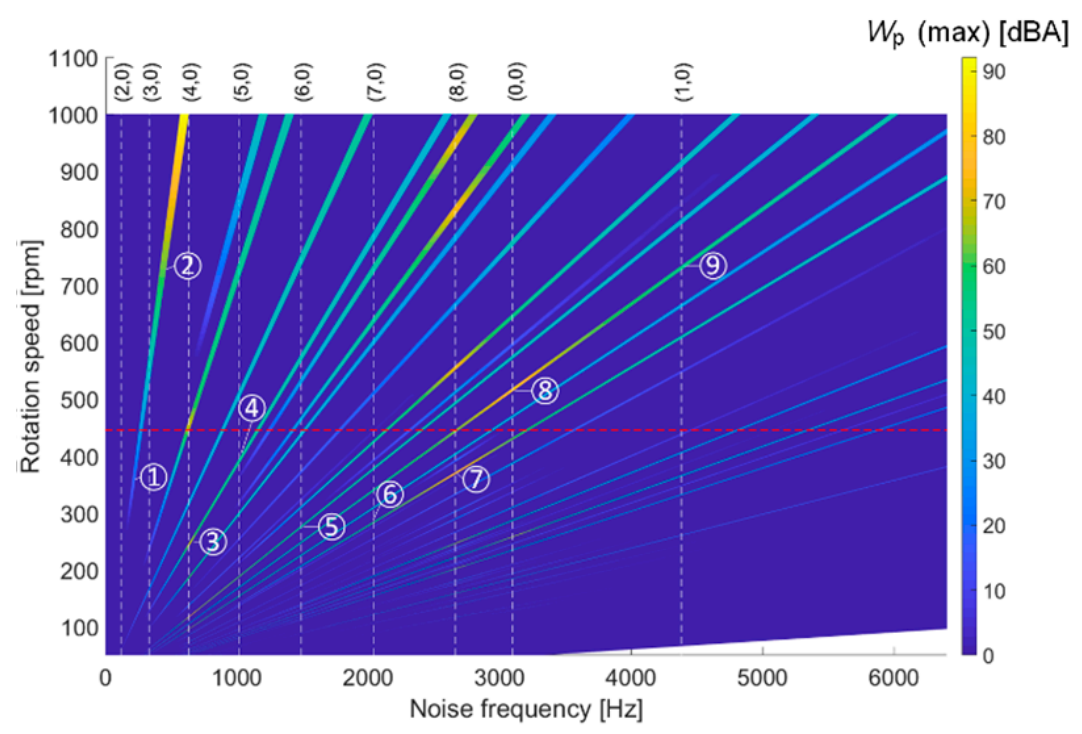

Figure 6. A-weighted maximum magnetic noise spectrogram of WHM-PMSM.

\section{FEA and Experimental Verifications}

The stator contains 36 segmented teeth because of closed slot modulation in order to reduce the amplitude of cogging torque significantly. The proposed equations in the previous section explain how the resulting magnetic flux density distribution by a MS-SDM and 3D FE model with variable range analysis, in which the maximum flux density is around the stator teeth and the outer surface of stator.

\section{Electromagnetic-Based Investigations}

Figure 7 presents a comparison among three different MS-SDM methods, in which the largest relative error is reported for the conventional MS-SDM, where several significant assumptions are taken, such as axial leakage of the field. However, the methodology is fast. The second method uses Gauss points for the slice distribution of the model. It offers the lowest relative errors at under 15 slices and higher error for the 15 and 20 slices. The proposed method employed uses 2D FEA in MS-SDM, which stores $B$ at each slice using FEA. This method shows a smaller relative error $\left(10^{-3}\right)$ in higher number of slices, such as 10 to 20 .

Figure 8 demonstrates a good agreement between MS-SDM and 3D-FEA models, where the harmonics as function of space (shown in Figure 8a) and time (Figure 8b) are compared. In Figure 8a, the largest harmonic exists on wavenumber 20 (as the WHM-PMSM has 20 pole pairs). As the saturation effect on the magnetic field is not considered by MS-SDM simulation, thus, there are very small differences presented in Figure 8a,b. The temporal harmonic contents on the flux is the same; however, the amplitude varies on the fundamental and the first harmonic. Indeed, the saturation is flattening the spatial distribution of magnetic flux such that the first harmonics are lower than the MS-SDM results. 


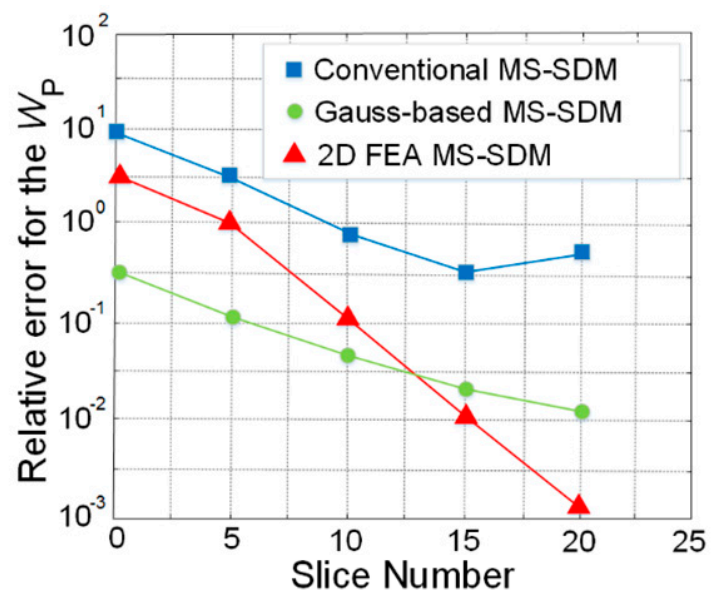

Figure 7. Convergence of the relative error on the maximum magnetic noise $W_{p}$ using different multi-slice subdomain models (MS-SDMs).
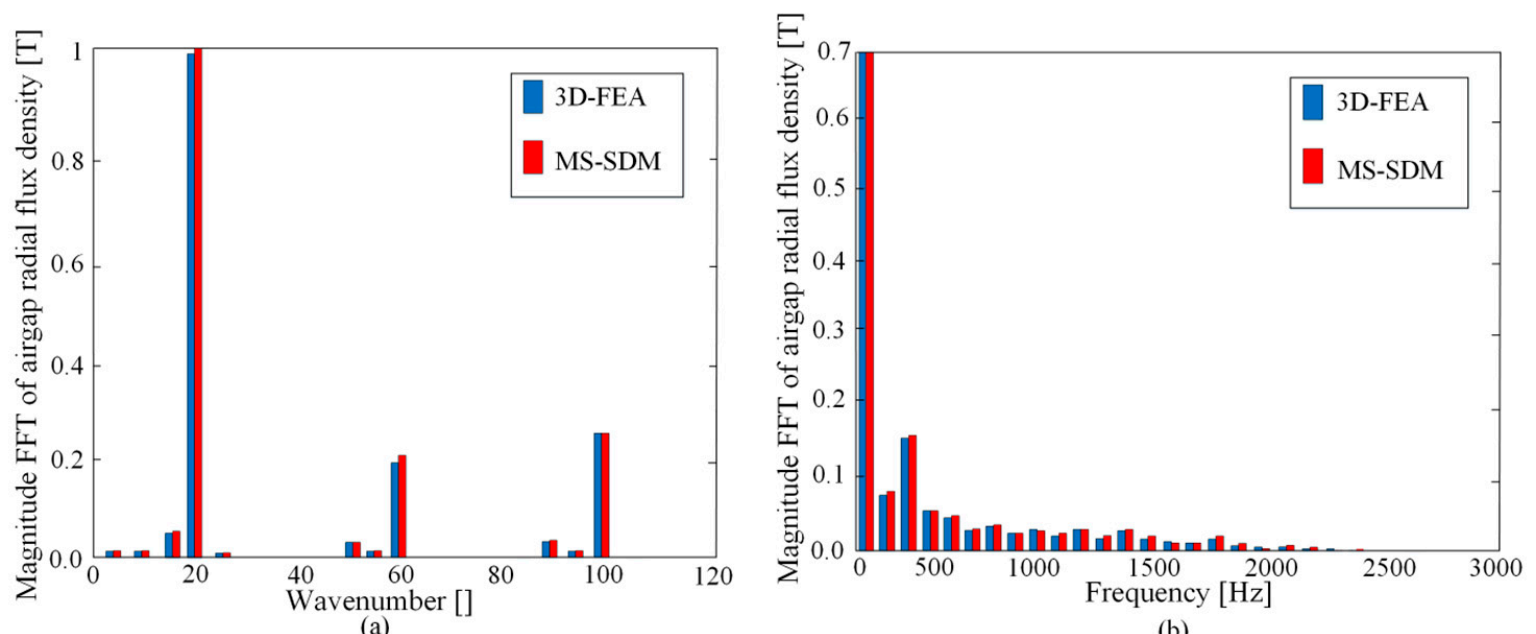

Figure 8. Magnitude fast fourier transform (FFT) of radial flux density using MS-SDM and 3D-FEA, as listed: (a) as function of space and (b) as function of time.

Figure 9 validates how the no-load electromagnetic main characteristics of the PMSM is varied between the initial model (ini) without skewing and optimized (opt) PMSM. Figure 9a demonstrates three-phase balanced back-EMF waveforms produced from flux linkage rate of change over time, in every individual coil. As presented, the optimized PMSM's back EMF is verified by experimental and FEA investigations. Figure $9 \mathrm{~b}$ presents the harmonic content of the back-EMF resulted from the fast Fourier transform analysis, it is shown that skewed PMSM model (opt) significantly reduced the harmonics mainly at higher orders, such as $5,7,9,11$, and 13 . The reduction of fundamental harmonic (first order) due to skewing is experimentally measured by 3.5\%. Although the peak-to-peak back-EMF is also slightly decreased, increasing the magnet thickness has compensated that loss which resulted in higher net magnet weight from 1.82 to $1.98 \mathrm{~kg}$. Figure $9 \mathrm{c}$ demonstrates how the skewing technique considerably quenches the peak-to-peak cogging torque for different rotor positions from 15 to 1.2 N.m. The electromagnetic instantaneous torque for initial and optimized PMSM (with 20 slices) is illustrated in Figure 9d, where the skewing has suppressed the peak torque production by approximately $11 \mathrm{~N} . \mathrm{m}$. The torque ripple $\left(T_{\max }-T_{\min } / T_{\mathrm{avg}} \times 100\right)$ is also ideally decreased, in which the initial and optimum PMSMs have 9\% and 7.9\%, respectively. In Figure 9e, the total loss as a function of slice number is provided, using MS-SDM and FEA simulations. The study reinforces that the slice number can reduce the total loss due to presence of high eddy-current loss at both iron cores and magnets. However, this modification comes with cost in the manufacturing stage; therefore, it is 
indeed an engineering trade-off among several factors, such as noise and vibration reduction and electromagnetic capability, as well as the manufacturing costs. In addition, the results show that the current phase angle affects the total core and magnet losses at certain armature current amplitude of 150A. The transient eddy-current loss produced at the magnets is simulated under different current angles of $60^{\circ}$ and $90^{\circ}$. Additionally, the simulations indicate that PWM converter and sinusoidal supply can highly influence the amplitude of the magnet loss because of the harmonics injected by the PWM converter. The FEA-based simulation and experimental results verify that the semi-analytical MS-SDM approach is reliable for electromagnetic capabilities (including noise and vibration) predictions.
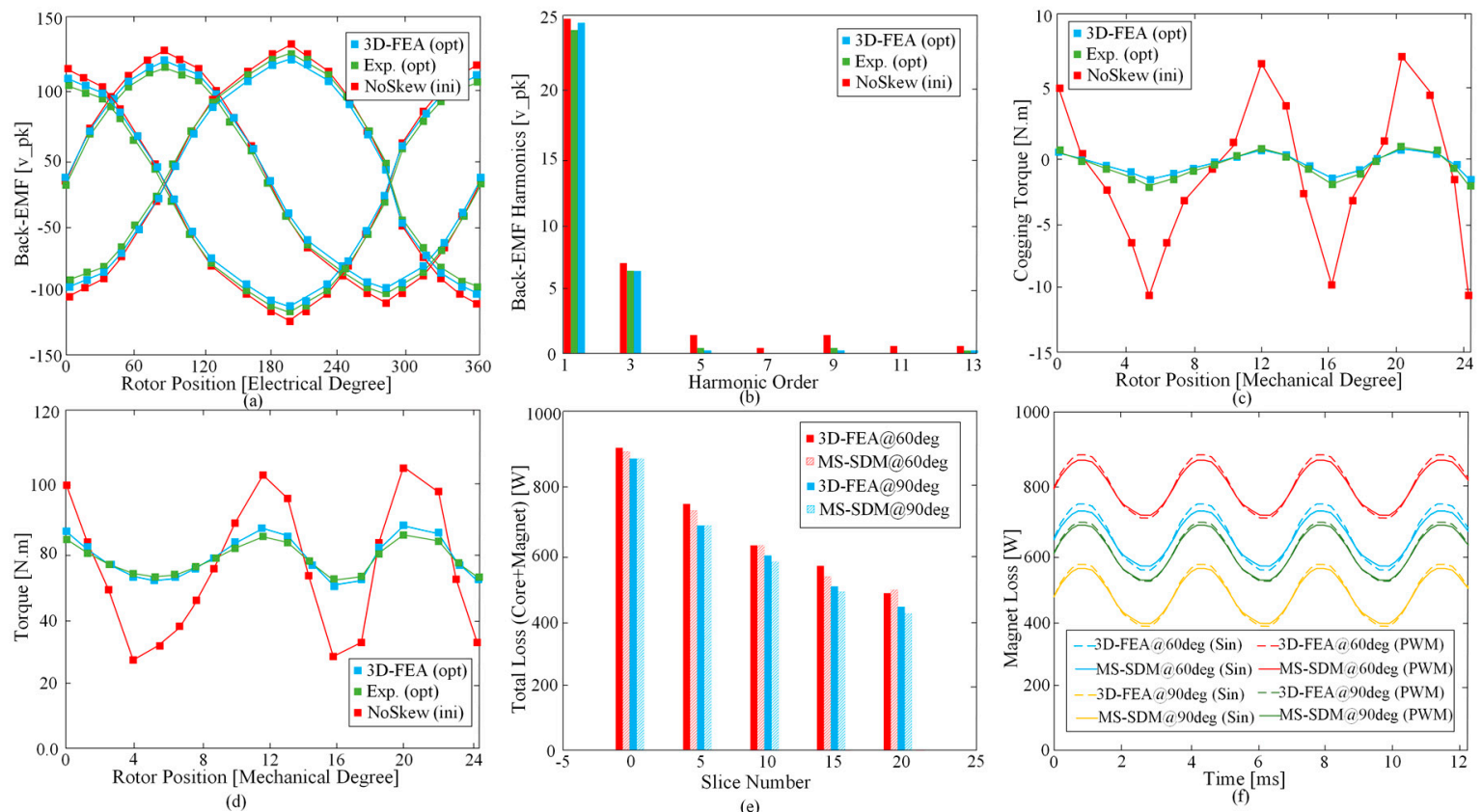

Figure 9. Electromagnetic capability validations of the in-wheel PMSM, listed as: (a) three-phase back-EMF, (b) critical back-EMF harmonics, (c) cogging torque (d) torque, (e) effect of iron core slice number on the total loss as function of different current phase angles, and (f) transient magnet loss.

A modular system of Brüel \& Kjær's sound power kit is used, which is detailed in Table 3, in which all component models are given.

Table 3. Modules of Brüel \& Kjær's sound power kit.

\begin{tabular}{ccc}
\hline Component & Model & Range \\
\hline PULSE Empty C-Size Front End (5In)-Dyn-X & $3560-C-E 12$ & - \\
\hline Microphone of $\frac{1}{2}$ (free field) & 4188 & $8-12.5 \mathrm{kHz}$, pre-polarized \\
\hline preamplifier microphone $\frac{1}{2}$ & $2669 \mathrm{~B}$ & - \\
\hline Microphone Cable LEMO & AO-0419-d-150 & 0 B to 1 B connector 15m \\
\hline windscreen of $\frac{1}{2}$ mod. UA0459 & EG-9999 & - \\
\hline PULSE Sound Power, Node-locked License & $7799-\mathrm{N}$ & - \\
\hline CPB analysis program and overall of 1 channel & $7771-\mathrm{N} 1$ & - \\
\hline program analysis of FFT and Overall of 1 channel & $7770-\mathrm{N} 1$ & - \\
\hline Sound level calibrator & 4231 & - \\
\hline
\end{tabular}

To gather noise data of the WHM-PMSM under $150 \mathrm{rpm}$ (rated speed), the PMSM was placed, based on ISO 6396. The microphone has been placed in radial direction of the rotor by $60 \mathrm{~cm}$ away 
from the PMSM to reduce the effect from the airflow, which are induced by the rotating rotor in an anechoic environment, which is shown in Figure 10. This device allows for measuring sounds and noises with various frequency filters. In this assessment, one-third octave band filters are used because of the following advantage: higher accuracy due to lower effect of the ambient sounds and those noises which are known as external sources to the main noise source being measured. In addition, the vibration meter provides the needed data by FFT analysis. A high-performance vector control module (PI-PWM) from OMRON with the CIMR-A model is used for this experiment.

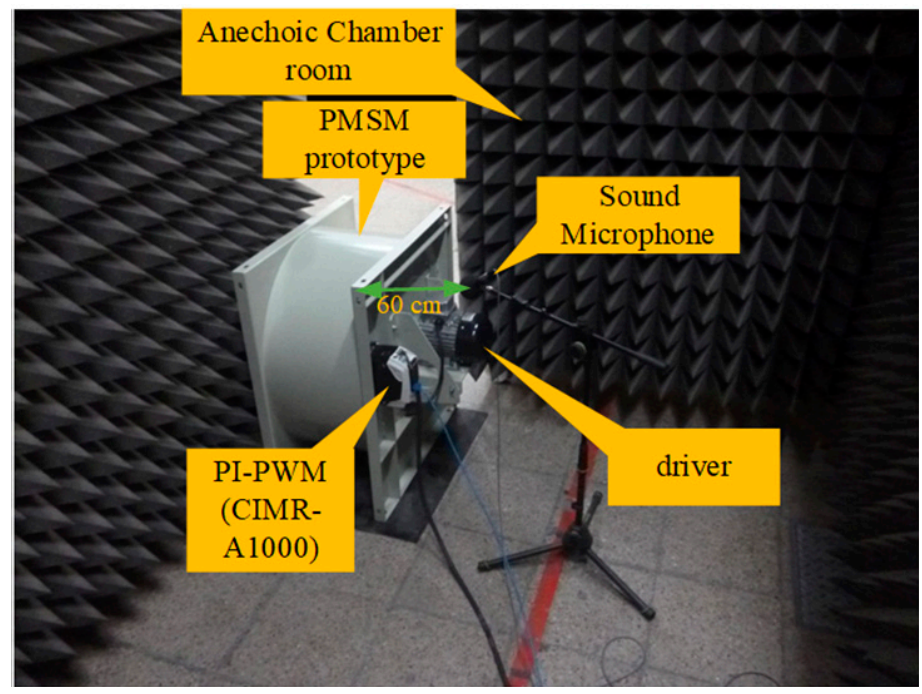

Figure 10. The maximum magnetic noise spectrogram of the optimum WHM-PMSM.

The manufactured prototype (shown in Figure 11) with the optimum skew angles was tested for $5 \mathrm{~kW}$ power consumption. Figure 11a,b presents the skewed magnets of the rotor and wounded stator core. Figure 11c indicates the assembled wheel hub-mounted PMSM in the front wheel of the vehicle. The motor was electromagnetically optimized in another recent study, which can be found in Reference [17].

A key feature is the Dyn-X technology, which avoids the need for input ranging via dual A/D converters to ensure you get the data the first time-when reaching an effective dynamic range of over $160 \mathrm{~dB}$. This equipment operates as sample-synchronous phase alignment between all channels using IEEE 1588v2 precision time protocol (PTP) and GPS time synchronization. The measurement has been made in an anechoic room in order to avoid any external source of noise.

During the vibration test, the following physical-based magnitudes are measures for the studied WHM-PMSM, where depending on what the aim of the measurement is, some may be more appropriate to be considered:

(a) Displacement (frequency range of below $100 \mathrm{~Hz}$ ).

(b) Speed (frequency range of $10 \mathrm{~Hz}$ to $1 \mathrm{kHz}$ ).

(c) Acceleration (high frequency measurement $>1 \mathrm{kHz}$ ).

The Rion vibration analyzer VA-12 allows precise vibration measurement by a piezoelectric accelerometer attached to a magnet. The meter provides an FFT analyzer measuring mode with both spectrum and time waveforms.

During the vibration test, the measuring instrument was set to provide an FFT analysis via acceleration measurements. The frequency span measured is over $5000 \mathrm{~Hz}$ to include high pass and low pass filters of $3 \mathrm{~Hz}$ and $20 \mathrm{kHz}$, respectively. Three measurement are done due to the $\mathrm{x}$-axis, $\mathrm{y}$-axis, and $z$-axis directions of vibration waves.

The presence of the magnetic noise produced in form of sound pressure level as a function of frequency is obtained at 15,32 , and $50 \mathrm{~Hz}$. 

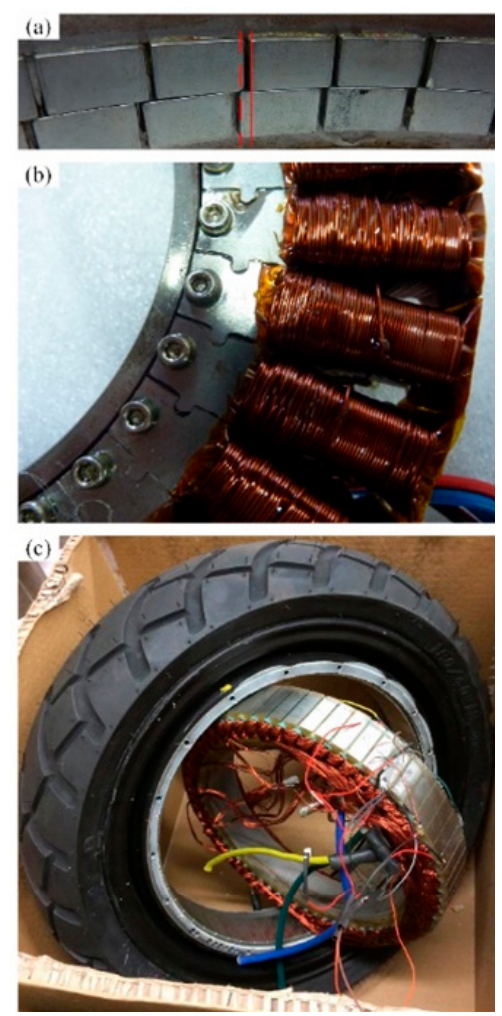

Figure 11. Manufactured WHM-PMSM based on optimized skew angles, listed as: (a) Permanent magnets including rotor iron back, (b) stator slots, (c) assembled wheel hub-mounted-PMSM.

Table 4 reports the influence of the different models with various values of skew angle and slice number on the electromagnetic capability of the PMSM. $W_{\text {vib,max }}$ shows the maximum magnitude of vibration in the defined speed range of 15-150 rpm, in which the vibration has reduced in the optimized model by $7.2 \mathrm{~dB}$ in comparison to initial model. Furthermore, small improvements can be reported for output power $P_{\mathrm{o}}$, electromagnetic torque $T$, efficiency $\eta$, and constant power speed range constant-power-speed-range (CPSR). In addition, the fundamental $d$-axis phase flux linkage $\lambda_{\mathrm{m}}$ remains almost constant.

Table 4. Electromagnetic results and verification of the optimal skew angles with 20 slices.

\begin{tabular}{ccccc}
\hline Parameters/Model & No Skew & Initial & Optimized & Test \\
\hline$\lambda_{\mathrm{m}}(\mathrm{mVs})$ & 1691.5 & 1714 & 1721.3 & 1718 \\
\hline$P_{\mathrm{o}}(\mathrm{W})$ & 449.2 & 503.2 & 514.7 & 511.7 \\
\hline$\eta(\%)$ & 92.1 & 95.8 & 97 & 96.9 \\
\hline CPSR & 434.5 & 477.3 & 487.9 & 505.8 \\
\hline Net Torque & 314.6 & 367.4 & 367.2 & 366.8 \\
\hline$L_{\text {vib, } \max }(\mathrm{dB})$ & 118.7 & 110.6 & 102.8 & 105.6 \\
\hline
\end{tabular}

Figure 11 illustrates A-weighted sound pressure level (ASPL) as function of rotating speed (up to $1000 \mathrm{rpm}$ ). The blue curve presents the proposed WHM-PMSM model with peak value $65.8 \mathrm{dBA}$ for speed range between 15-150 rpm, which has been experimentally verified by $78.6 \mathrm{dBA}$ which is shown in the red color for the optimum skew angles and slice. In addition to the predicted and measured optimized model, the predicated initial model (in orange color) based on the empirical model is presented to highlight the improvement, where the peak radiated ASPL is 79.7 dBA under ratings operation. Note that the maximum ASPL over rating speed is above approximately $10 \mathrm{dBA}$. 
During the test, the rotor of the studied PMSM was coupled to an induction motor for deriving purpose, the induction motor itself produced between 5 to $15 \mathrm{dBA}$ (these values are measured when the induction motor was operating without coupling with other machines) between 0-1000 rpm. Therefore, the error between predicted and measured curves is less than 3\%. Two of the in-wheel motors used have the feature of generating regenerative power during the braking, therefore, the driver was employed to observe the regenerative mode. While the proposed WHM-PMSM was tested the driver was in open-circuit condition because of its sole positive acceleration (motoring) characteristic.

The experimental measurement was conducted in the laboratory of Electrical Machines and Drives at Universitat Politècnica de Catalunya-Barcelona Tech. The environmental impact was carefully considered. In fact, noise pollution persistency in any form of vibrations and audible noise in residential or industrial ranges are harmful and can even cause health issues to anyone who is near the sources.

\section{Conclusions}

A light-duty electric vehicle equipped with four in-wheel hub-mounted PMSMs, in which even integer slot number (in this case 36) has empirically resulted in only even force wavenumbers (0, 2, or 4) of Maxwell force harmonics, which literally avoids an unbalanced magnetic force (the net electromagnetic force in the stator). However, all the wavenumbers should be considered via a variable-speed computation of electromagnetically-excited noise to give a precise prediction of the vibro-acoustic outcome. In this research, the audible magnetic noise radiated under different angles of both stator and rotor skews were examined for a variable-speed analysis using MS-SDM to find the best skew angle and slice number, which minimizes the sound power, while the electromagnetic capability of the machine remains constant. The impact of the motor's natural frequencies was also considered to avoid resonance when the motor operates under defined ratings. For considering all the possible resonances which might occur in this WHM-PMSM with $(5-50 \mathrm{~Hz})$, the variable speed range of over $5000 \mathrm{~Hz}$ vibration frequency has been used and has been presented in the acoustic sonograms. Finally, the WHM-PMSM with optimal skew angles and slice number have reduced the maximum sound pressure and power levels by $10 \mathrm{dBA}$ and $5 \mathrm{dBA}$ (in average), when the motor operates under ratings (150 rpm in this case). Due to lower force harmonics, this improvement is reached by the optimum skew angles in the stator and rotor cores. Additionally, the skew slice number of 20 was chosen due to its contribution on the net electromagnetic force, which indirectly has small effect on the resulted sound power, approximately less than $1 \mathrm{dBA}$. From a manufacturing point of view, the slice number of 20 is expensive, and the prototyped WHM-PMSM is manufactured using 20 slices to validate the e-NVH numerical achievements. The study also presented that a slice number between 10 to 15 offers a reasonable reduction of e-NVH with an acceptable relative error, in addition to an inexpensive manufacturing of both stator and rotor parts. The choice of slice number is a trade-off between cost and electromagnetic benefits that, depending on the application requirements, can be tolerated. For traction motors, the overall recommendations of the study are the skewing angle of $12^{\circ}$ and $8^{\circ}$ for rotor and stator cores, and a slice number between 5 to 15 are suggested. All the computations have been numerically (3D-FEA) and experimentally verified through magnetic noise measurements with a small error.

Author Contributions: Investigation, Methodology, Software, Visualization, Writing, Measurement, Validation, P.A.; Software, Supervision, Writing-Review and Editing, Project Director, R.B.; Supervision, Review and Editing, A.L. All authors have read and agreed to the published version of the manuscript.

Funding: This research received no external funding.

Acknowledgments: This work was supported by Energy Processing and Integrated Circuits Group (EPIC) of the Universitat Politècnica de Catalunya-BarcelonaTech.

Conflicts of Interest: The authors declare no conflict of interest. 


\section{References}

1. El-Refaie, A.M. Fractional-slot concentrated-windings synchronous permanent magnet machines: Opportunities and challenges. IEEE Trans. Ind. Electron. 2010, 57, 107-121. [CrossRef]

2. Tessarolo, A.; Luise, F.; Pieri, S.; Benedetti, A.; Bortolozzi, M.; de Martin, M. Design for manufacturability of an Off-Shore Direct-Drive wind generator: An insight into additional loss prediction and mitigation. IEEE Trans. Ind. Appl. 2017, 53, 4831-4842. [CrossRef]

3. El-Refaie, A.M.; Jahns, T.M. Scalability of surface PM Machines with concentrated windings designed to achieve wide speed ranges of constant-power operation. IEEE Trans. Energy Convers. 2006, 21, 362-369. [CrossRef]

4. Ahsanullah, K.; Dutta, R.; Rahaman, M.F.; Jahns, T.M. Analysis of Low-Speed IPMMs with Distributed and Fractional Slot Concentrated Windings for Wind Energy Applications. IEEE Trans. Magn. 2017, 53, 1-10. [CrossRef]

5. Wu, L.; Qu, R.; Li, D. Reduction of Rotor Eddy-Current Losses for Surface PM Machines with Fractional Slot Concentrated Windings and Retaining Sleeve. IEEE Trans. Magn. 2014, 50, 1-4. [CrossRef]

6. Valavi, M.; Nysveen, A.; Nilssen, R.; Lorenz, R.D.; Rølvåg, T. Influence of pole and slot combinations on magnetic forces and vibration in low-speed PM wind generators. IEEE Trans. Magn. 2014, 50, 1-4. [CrossRef]

7. Zhu, Z.Q.; Xia, Z.P.; Wu, L.J.; Jewell, G.W. Analytical modeling and finite-element computation of radial vibration force in fractional-slot permanent-magnet brushless machines. IEEE Trans. Ind. Appl. 2010, 46, 1908-1918. [CrossRef]

8. Chen, Y.S.; Zhu, Z.Q.; Howe, D. Vibration of PM brushless machines having a fractional number of slots per pole. IEEE Trans. Magn. 2006, 42, 3395-3397. [CrossRef]

9. Okuyama, Y.; Moriyasu, S. Electromagnetic noise of induction motors driven by PWM inverters. Electr. Eng. Jpn. 2000, 133, 55-62. [CrossRef]

10. Hubert, A.; Frienrich, G. Influence of power converter on induction motor acoustic noise: Interaction between control strategy and mechanical structure. IEE Proc. Electr. Power Appl. 2002, 149, 93-100. [CrossRef]

11. Le Besnerais, J.; Lanfranchi, V.; Hecquet, M.; Brochet, P. Multiobjective Optimization of Induction Machines Including Mixed Variables and Noise Minimization. IEEE Trans. Magn. 2008, 44, 1102-1105. [CrossRef]

12. Le Besnerais, J.; Lanfranchi, V.; Hecquet, M.; Brochet, P. Optimal Slot Numbers for Magnetic Noise Reduction in Variable-Speed Induction Motors. IEEE Trans. Magn. 2009, 45, 3131-3136. [CrossRef]

13. Xia, C.; Zhang, Z.; Geng, Q. Analytical Modeling and Analysis of Surface Mounted Permanent Magnet Machines with Skewed Slots. IEEE Trans. Magn. 2015, 51, 1-8.

14. De Gersem, H.; Hameyer, K.; Weiland, T. Skew Interface Conditions in 2-D Finite-Element Machine Models. IEEE Trans. Magn. 2003, 39, 1452-1455. [CrossRef]

15. Le Besnerais, J.; Lanfranchi, V.; Hecquet, M.; Brochet, P. Characterization and Reduction of Audible Magnetic Noise Due to PWM Supply in Induction Machines. IEEE Trans. Ind. Electron. 2010, 57, 1288-1295. [CrossRef]

16. Le Besnerais, J.; Lanfranchi, V.; Hecquet, M.; Friedrich, G.; Brochet, P. Characterisation of radial vibration force and vibration behaviour of a pulse-width modulation-fed fractional-slot induction machine. IET Electr. Power Appl. 2009, 3, 197-208. [CrossRef]

17. Devillers, E.; le Besnerais, J.; Lubin, T.; Hecquet, M.; Lecointe, J.P. A review of subdomain modeling techniques in electrical machines: Performances and applications. In Proceedings of the IEEE ICEM International Conference on Electrical Machines, Lausanne, Switzerland, 4-7 September 2016.

18. Lin, F.; Zuo, S.; Deng, W.; Wu, S. Modeling and Analysis of Acoustic Noise in External Rotor In-Wheel Motor Considering Doppler Effect. IEEE Trans. Ind. Electron. 2018, 65, 4524-4533. [CrossRef]

19. Kotter, P.; Morisco, D.; Boesing, M.; Zirn, O.; Wegener, K. Noise-Vibration-Harshness-Modeling and Analysis of a Permanent-Magnetic Disc Rotor Axial-Flux Electric Motor. IEEE Trans. Magn. 2018, 54, 1-4. [CrossRef]

20. Nobahari, A.; Darabi, A.; Hassannia, A. Various skewing arrangements and relative position of dual rotor of an axial flux induction motor, modelling and performance evaluation. IET Electr. Power Appl. 2018, 12, 575-580. [CrossRef]

21. Lee, C.M.; Seol, H.S.; Lee, J.Y.; Lee, S.H.; Kang, D.W. Optimization of Vibration and Noise Characteristics of Skewed Permanent Brushless Direct Current Motor. IEEE Trans. Magn. 2017, 53, 1-5. [CrossRef]

22. Xu, W.; Bao, X.; Di, C.; Wang, L.; Chen, Y. Optimal Angle Combination for Improving Electromagnetic Torque in Induction Motor With Double-Skewed Rotor. IEEE Trans. Magn. 2017, 53, 1-5. [CrossRef] 
23. Kang, C.H.; Kang, K.J.; Song, J.Y.; Cho, Y.J.; Jang, G.H. Axial Unbalanced Magnetic Force in a Permanent Magnet Motor Due to a Skewed Magnet and Rotor Eccentricities. IEEE Trans. Magn. 2017, 53, 1-5. [CrossRef]

24. Wang, L.; Bao, X.; Di, C.; Li, J. Effects of Novel Skewed Rotor in Squirrel-Cage Induction Motor on Electromagnetic Force. IEEE Trans. Magn. 2015, 51, 1-5. [CrossRef]

25. Wang, C.; Bao, X.; Xu, S.; Zhou, Y.; Xu, W.; Chen, Y. Effects of Novel Skewed Rotor in Squirrel-Cage Induction Motor on Electromagnetic Force. IEEE Trans. Magn. 2017, 53, 1-5. [CrossRef]

26. Min, S.G.; Sarlioglu, B. Modeling and Investigation on Electromagnetic Noise in PM Motors with Singleand Double-Layer Concentrated Winding for EV and HEV Application. IEEE Trans. Transp. Electrif. 2018, 4, 292-302. [CrossRef]

(C) 2020 by the authors. Licensee MDPI, Basel, Switzerland. This article is an open access article distributed under the terms and conditions of the Creative Commons Attribution (CC BY) license (http://creativecommons.org/licenses/by/4.0/). 